\title{
Bioestimulação da Atividade Reprodutiva de Coelhas Lactantes em Regime de Monta Natural ${ }^{1}$
}

\section{Ana Silvia Alves Meira Tavares Moura², Simone Fernandes ${ }^{3}$, José Luiz Moraes Vasconcelos ${ }^{4}$, Elaine Bianospino 5}

\begin{abstract}
RESUMO -Esta pesquisa avaliou o efeito da separação mãe-ninhada sobre o desempenho reprodutivo de coelhas lactantes e ponderal das ninhadas até aos 70 dias de idade. Examinaram-se 415 exposições ao macho de 157 fêmeas lactantes distribuídas em dois grupos: controle, com livre acesso ao ninho, e tratado, cujo acesso bloqueou-se entre o sétimo e o nono dias pós-parto, durante as 39 horas que precederam a cobrição. Das 415 exposições ao macho, 387 resultaram em cobrições e 264 em partos. As fêmeas tratadas, de quinta lactação em diante, apresentaram receptividade e taxa de parição, respectivamente, 6,7\% e 10,8\% maiores em comparação às fêmeas do grupo controle. Não se constatou efeito da interação tratamento x ordem de lactação, ou do tratamento, com respeito ao número de láparos/ ninhada à desmama e nos 70 dias de idade. Em ninhadas de primíparas, a taxa de mortalidade da separação até 70 dias de idade foi maior no grupo tratado, em relação ao controle $(0,248 \pm 0,047$ vs. $0,120 \pm 0,047)$. O peso total da ninhada à desmama foi inferior no grupo tratado, do que no controle $(5.753 \pm 93 \mathrm{~g}$ vs. $6.222 \pm 89 \mathrm{~g})$ em todas as ordens. Todavia, não se observou diferença no peso total ou médio da ninhada nos 70 dias de idade. O tratamento também reduziu o peso individual à desmama $(912,4 \pm 4,9 \mathrm{~g}$ vs. $962,7 \pm 4,5 \mathrm{~g})$ em todas as ordens de lactação, entretanto, elevou o ganho de peso médio diário pós-desmama $(32,25 \pm 0,19 \mathrm{~g} / \mathrm{d}$ vs. 31,44 $\pm 0,17 \mathrm{~g} / \mathrm{d})$, proporcionando a ocorrência de ganho compensatório neste período. Pode-se recomendar a separação temporária mãe-ninhada para melhorar o desempenho reprodutivo de coelhas lactantes multíparas; mas deve ser evitada para as primíparas, devido à elevação da taxa de mortalidade das ninhadas.
\end{abstract}

Palavras-chave: bioestimulação, coelhos, desempenho de ninhadas, manejo reprodutivo, receptividade sexual, taxa de parição

\section{Bioestimulation of the Reproductive Activity of Lactating Rabbit Does in a Natural Mating System}

\begin{abstract}
The objective was to evaluate the effect of temporary mother-litter separation on the reproductive performance of lactating does and growth performance of their litters up to 70 days of age. A total of 415 presentations of 157 lactating does assigned to two groups: control, with free nest access, and treated, whose nest access was closed during the 39 hours that preceded mating, were analyzed. From the 415 presentations, 387 resulted in matings and 264 in parturitions. Does of the treated group, from fifth and further lactations, had sexual receptivity and parturition rate, respectively, $6.7 \%$ and $10.8 \%$ higher than those females of the control group. No treatment by lactation order interaction, or treatment effects, were detected on number weaned and number of young per litter at 70 days. In litters of primiparous does, mortality rate from separation up to 70 days of age was higher in the treated group ( $0.248 \pm 0.047$ vs. $0.120 \pm 0.047)$. Litter weaning weight was lower in the treated group compared to the control group $(5,753 \pm 93 \mathrm{~g}$ vs. $6,222 \pm 89 \mathrm{~g})$, for all lactation orders, but no differences were detected in total and average 70-d weights between groups. Treatment also reduced individual weaning weight $(912.4 \pm 4.9 \mathrm{~g}$ vs. $962.7 \pm 4.5 \mathrm{~g})$ for all lactation orders, but increased postweaning average daily gain $(32.25 \pm 0.19 \mathrm{~g} / \mathrm{d}$ vs. $31.44 \pm 0.17 \mathrm{~g} / \mathrm{d}$ ), favoring the occurrence of a compensatory weight gain in this period. Temporary mother-litter separation may be recommended to improve reproductive performance of lactating multiparous does, but should be avoided for primiparous due to the increased mortality rate of their litters.
\end{abstract}

Key Words: bioestimulation, litter performance, parturition rate, rabbits, reproductive management, sexual receptivity

\section{Introdução}

É crescente a demanda de carne de coelhos como alternativa saudável à alimentação do homem. Dentre suas qualidades dietéticas destacam-se: a cor branca, a proteína de alto valor biológico e, sobretudo, o baixo teor de gordura predominante nos ácidos graxos insaturados (Russo et al., 1998). Avoluma, igualmente, a procura de coelhos para modelos experimentais em diversas áreas da pesquisa biomédica. Apesar da demanda notória, observa-se baixo índice de produtividade na cunicultura nacional, em conseqüência do

${ }^{1}$ Projeto financiado pela FAPESP.

2 Profa. Dra. DPEA/FMVZ/UNESP, Botucatu-SP, CEP: 18618-000. E.mail: anamoura@fca.unesp.br

3 Zootecnista, MSc., DPEA/FMVZ/UNESP, Botucatu-SP.

4 Prof. Dr. DPEA/FMVZ/UNESP, Botucatu-SP. E.mail: vasconcelos@fca.unesp.br

${ }^{5}$ Aluna de Pós-graduação em Nutrição e Produção Animal, FMVZ/UNESP, Botucatu, SP. 
atraso tecnológico em que se encontra. Uma das causas desse atraso é o manejo reprodutivo do rebanho, que tem evoluído rapidamente para o agrupamento de tarefas e ciclização da produção em países de cunicultura avançada, em oposição ao manejo individual aqui praticado, em granjas comerciais ou em biotérios.

Embora a coelha apresente ovulação induzida pelo coito, a sincronização das cobrições constitui o principal entrave à ciclização da produção. Isso, por estar condicionada à receptividade sexual variável, especialmente em coelhas lactantes. O método mais empregado para sincronizar coelhas submetidas à inseminação artificial é a aplicação de gonadotrofina sérica de égua prenhe (PMSG) dois ou três dias antes da inseminação, sem considerar, todavia, a receptividade sexual (Boiti et al., 1995). Mas o uso generalizado de PMSG conduz à disfunção ovariana e imunização das coelhas, após sucessivas aplicações (Maertens et al., 1995). Soma-se a isto, a necessidade de manter a imagem de produto "natural" junto aos consumidores, reduzindo-se, ou mesmo evitando-se, o uso de gonadotrofinas em cunicultura (Castellini, 1996).

Métodos alternativos, denominados bioestimulatórios, vêm sendo encorajados pelo International Rabbit Reproduction Group (Boiti, 1998) com o propósito de melhorar a receptividade sexual de coelhas nulíparas e lactantes. A bioestimulação consiste no emprego de fator estressante para alterar o equilíbrio endócrino da fêmea, e melhorar os resultados reprodutivos sem a utilização de hormônios (Castellini, 1995). As técnicas sugeridas abrangem: a adoção de programas nutricionais e de luz, a manipulação da fêmea e a separação temporária entre fêmea e ninhada (Luizi \& Crimella, 1998; Maertens, 1998). O efeito benéfico do fotoperíodo constante durante todo o ano com respeito à receptividade sexual das coelhas foi demonstrado experimentalmente (Walter et al., 1968). Dentre os demais métodos, o melhor e mais promissor, tanto pela viabilidade prática em larga escala, como pelos resultados obtidos até o momento, é a separação temporária entre mãe e ninhada, aos quatro ou nove dias de idade, por período de 24 a 48 horas (Theau-Clement \& Boiti, 1998). Sabendo-se que naturalmente a coelha amamenta sua ninhada uma vez a cada 24 horas (Lebas et al., 1996), na prática esta medida implica em privar a ninhada de uma mamada.

Para coelhas no quarto dia de lactação, 24 horas de separação foram suficientes para aumentar a fertilidade, mas, no nono dia de lactação, 36 horas foram necessárias (Alvariño et al., 1998). Observase que, a partir de 24 horas de separação, o peso médio dos láparos à desmama é menor, embora a taxa de mortalidade pré-desmame não se altere (Alvariño et al., 1998; Maertens, 1998). Não se sabe, no entanto, se o efeito negativo sobre o peso à desmama, pode ser compensado após a desmama.

Os objetivos foram investigar se a separação temporária mãe-ninhada melhora o desempenho reprodutivo de coelhas lactantes, em regime de monta natural, e avaliar seu efeito sobre o desempenho ponderal das ninhadas até a desmama e inclusive nos 70 dias de idade.

\section{Material e Métodos}

\section{Animais e Manejo}

O experimento foi conduzido de fevereiro a novembro de 2000 na Área de Produção de Coelhos da Faculdade de Medicina Veterinária e Zootecnia da UNESP, Câmpus de Botucatu, em coelhos do grupo genético Botucatu. A população originou-se de coelhos Norfolk 2000, importados da Inglaterra pela Faculdade em 1971, que possuem cerca de 25 gerações de adaptação local. Incluem-se três anos de seleção para características de desempenho individual e de ninhadas (Moura \& Polastre, 2000). O plantel contemporâneo é constituído de 110 fêmeas e 20 machos reprodutores. São alojados em gaiolas de arame galvanizado, equipadas com comedouro, bebedouro automático do tipo chupeta e ninho de madeira só para as fêmeas. As gaiolas estão dispostas no sistema flat-deck no galpão-maternidade aberto, que possui telas metálicas nas laterais e orientação lesteoeste. $\mathrm{O}$ controle da ventilação processa-se através de cortinas plásticas reguláveis. O galpão de crescimento possui estrutura semelhante. Na maternidade adotou-se programa de luz constante, 14 horas de luz/ 24h (14L/10E), com o objetivo de minimizar os efeitos da variação natural do fotoperíodo sobre a receptividade das fêmeas reprodutoras. A iluminação foi realizada com 22 lâmpadas incandescentes de $100 \mathrm{~W}, 11$ em cada um dos dois corredores centrais, distanciadas longitudinalmente dois metros uma da outra. Considerando-se que a área total ocupada pelas gaiolas e corredores de circulação é de $176 \mathrm{~m}^{2}$ (22 $\times 8 \mathrm{~m})$, calculou-se a intensidade luminosa média, de $12,5 \mathrm{~W} / \mathrm{m}^{2}$. No galpão de crescimento, a ilumina- 
ção foi natural durante toda a fase experimental.

A ração peletizada, oferecida à vontade aos animais durante o experimento, foi produzida na fábrica de rações da Faculdade de Medicina Veterinária e Zootecnia da UNESP, Botucatu, a partir de fórmula constante à base de milho, farelo de soja, feno de Brachiaria decumbens e suplementos minerais e vitamínicos, contendo, aproximadamente (valores calculados), $18 \%$ de Proteína Bruta, $12 \%$ de Fibra Bruta, $16,5 \%$ de Fibra Detergente Ácido, $1,1 \%$ de Cálcio, $0,8 \%$ de Fósforo e $2.700 \mathrm{kcal} / \mathrm{kg}$ de Energia Digestível na matéria natural.

No rebanho, as fêmeas iniciavam a vida reprodutiva entre quatro e cinco meses de idade, e os machos, entre cinco e seis meses. O período partocobrição variou de nove a 11 dias, na dependência do dia da semana em que ocorreu a parição. Todas as cobrições foram realizadas em dia fixo da semana, às sextas-feiras pela manhã. Imediatamente antes de se transportar a fêmea até a gaiola do macho, registrouse a coloração dos lábios vulvares. Estando receptiva, o coito ocorreu em cinco minutos; caso contrário, foi registrada a recusa. Cada um dos 20 machos recebeu uma ou duas fêmeas para a cobrição, efetuando, no máximo, dois saltos num mesmo dia. Realizou-se o diagnóstico de gestação 10 dias após a cobrição, através de palpação ventral. O ninho foi posto na gaiola da fêmea no $28^{\circ}$ dia de gestação e retirado na quarta semana pós-parto. Os critérios para descarte de fêmeas reprodutoras incluíram a ocorrência de três recusas ao macho ou três palpações negativas consecutivas; os de machos reprodutores, a redução da libido e da fertilidade. Esta última foi testada, mensalmente, através da comparação entre a taxa de prenhez das fêmeas servidas por um determinado macho e a taxa de prenhez média do rebanho. Machos e fêmeas eram descartados, se fossem notadas alterações no estado sanitário tais como: calos, abcessos e infecções respiratórias associadas à perda acentuada de peso. Reprodutores descartados, machos e fêmeas, foram imediatamente substituídos por animais jovens, aptos a iniciar a reprodução.

$\mathrm{O}$ registro de nascimentos ocorreu nas primeiras 16 horas após o parto. Já o desmame executouse no período de 34 a 36 dias de idade, ocasião em que os láparos foram pesados, identificados e alojados, oito por gaiola de $0,48 \mathrm{~m}^{2}$, no galpão de crescimento. Seu peso foi novamente auferido entre 69 e 71 dias de idade.

\section{Protocolo experimental}

R. Bras. Zootec., v.32, n.2, p.315-324, 2003
A separação temporária entre fêmea lactante e ninhada, 39 horas antes da apresentação ao macho para a cobrição, foi avaliada como método bioestimulatório da receptividade sexual. Inicialmente, 110 fêmeas nulíparas, primíparas ou multíparas foram, a priori, e aleatoriamente, distribuídas pelos dois grupos experimentais, tratado (bioestimulado) ou controle. Cada fêmea permaneceu no mesmo grupo durante todo o período experimental ou, então, até ser descartada. Foram registradas informações de coelhas em lactação, que possuíam pelo menos quatro láparos lactentes, dois dias antes da apresentação ao macho para a cobrição. $\mathrm{O}$ acesso das fêmeas do grupo bioestimulado ao ninho foi bloqueado por intermédio da interposição de quadros de madeira e tela metálica, às $16 \mathrm{~h}$ de quarta-feira, sete a nove dias pósparto. Os ninhos permaneceram, assim, bloqueados, durante todo o dia de quinta-feira. Na manhã de sexta-feira, às $7 \mathrm{~h}$, a tela foi removida permitindo o acesso da fêmea ao ninho e a amamentação da ninhada. Logo em seguida, a fêmea foi levada ao macho. As fêmeas do grupo controle tiveram livre acesso ao ninho.

\section{Análises estatísticas}

A coloração da vulva (pálida, rosada, vermelha ou violácea), a receptividade sexual (porcentagem de fêmeas receptivas em relação ao número de fêmeas expostas ao macho), e a taxa de parição (porcentagem de partos em relação ao número de fêmeas expostas ao macho) foram as características reprodutivas analisadas. Da ninhada seguinte, avaliaram-se: o número de nascidos totais e vivos, o peso total da ninhada ao nascer (láparos vivos) e taxa de natimortalidade. Quanto ao desempenho da ninhada separada (ou não) da mãe, examinou-se o número de láparos vivos à desmama e nos 70 dias de idade; os pesos total e médio da ninhada no desmame e nos 70 dias de idade; e as taxas de mortalidade da separação até a desmama e da separação até os 70 dias de idade. Adicionalmente, avaliou-se o desempenho individual destes láparos à desmama e nos 70 dias de idade, estudando-se o peso individual e o ganho de peso no período.

Os resultados foram agrupados em três categorias, de acordo com a ordem de lactação: primeira, segunda à quarta e quinta e demais. Para as variáveis discretas utilizou-se o teste de qui-quadrado, por categoria de ordem de lactação. Para as demais, o delineamento experimental em parcelas subdivididas 
(Sampaio, 1998). O modelo estatístico incluiu os efeitos fixos de tratamento, de mês de cobrição, de fêmea dentro de tratamento, de ordem de lactação e interações. Para as características de desempenho à desmama, adotaram-se os ajustes lineares para número de nascidos e idade à desmama. Para as características de desempenho aos 70 dias procederam-se ajustes lineares para número de lactentes, no momento da separação, e idade da pesagem. As interações não significativas foram excluídas dos modelos finais de análise. Os procedimentos FREQ/ CHISQ, CATMOD e GLM do PC-SAS (1996) auxiliaram as análises estatísticas.

\section{Resultados e Discussão}

Os resultados referentes à coloração da vulva, no momento da cobrição, são exibidos na Figura 1. Detectou-se efeito do tratamento sobre ela, no momento da cobrição, com maior incidência de vulva vermelha $(70,2$ vs. $44,1 \%)$ e menor de vulva rosada (15,4 vs. 37,4\%) nas fêmeas bioestimuladas, em relação às controle $(\mathrm{P}<0,01)$, considerando-se todas as ordens de parição em conjunto. A coloração e a turgidez da vulva são sinais importantes de receptividade sexual em coelhas. A receptividade foi maior (98\%) entre as fêmeas apresentadoras de vulva vermelha e menor (38\%), entre as de vulva pálida. Estes valores estão próximos dos apontados por Alvariño \& Ubilla (1993) de 97,5\% de

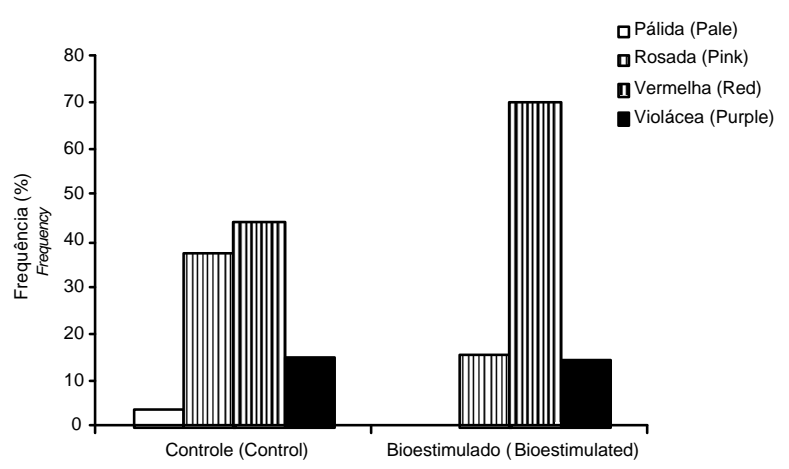

Figura 1 - Coloração da vulva em coelhas dos grupos controle e bioestimulado imediatamente antes da exposição ao macho.

Figure 1 - Color of vulva in rabbit does of the control and bioestimulated groups right before exposition to the buck. receptividade em fêmeas com vulva vermelha e 20,6\% com pálida. Segundo Maertens (1998), as proporções de coelhas lactantes demonstradoras de vulvas vermelhas e violáceas em conjunto, no momento da inseminação, foram de 78,8 e 40,8\% para os grupos bioestimulado (40 horas de separação da ninhada) e controle, respectivamente, no décimo primeiro dia pósparto. No presente estudo registraram-se valores mais elevados de 84,6 e $58,8 \%$, respectivamente, nos mesmos grupos.

Do total de 415 exposições de fêmeas ao macho realizadas a partir de 4 de fevereiro a 29 de setembro de 2000, 387 resultaram em cobrições e $264(63,6 \%$ das fêmeas expostas ao macho ou 68,2\% das fêmeas receptivas) em partos. Atribui-se a elevada receptividade sexual das fêmeas $(93,3 \%)$, tanto do grupo bioestimulado como controle (Tabela 1), pelo menos em parte, ao efeito favorável do fotoperíodo constante, com 14L:10E, propiciado pelo programa de suplementação artificial de luz (Theau-Clement et al., 1998). A opção de apresentar taxa de parição expressa em relação ao número de fêmeas expostas (e não ao número de fêmeas receptivas) ao macho, deve-se ao fato de que este índice reflete melhor a eficiência global do sistema em questão, embora resulte em valores absolutos mais baixos. Quando calculadas com base no número de fêmeas receptivas, as taxas de parição nos grupos controle e bioestimulado foram, respectivamente: 65,6 e $60,7 \%$ na primeira, 75,0 e $74,3 \%$ da segunda à quarta e 64,1 e 70,6\% a partir da quinta lactação.

Não se detectaram diferenças importantes entre fêmeas tratadas e controle da primeira e da segunda à quarta lactações, quanto à receptividade sexual e à taxa de parição (Tabela 1). Os resultados indicam que o efeito favorável do tratamento se manifesta com o avanço da vida reprodutiva, pois, somente para a categoria da quinta lactação em diante, as fêmeas tratadas apresentaram receptividade $6,7 \%$ superior à do grupo controle $(\mathrm{P}<0,05)$ e próxima de $99 \%$. No caso da taxa de parição, a diferença entre os grupos foi numericamente maior $(10,8 \% ; \mathrm{P}=0,13)$ para a mesma categoria. Considerando-se que ela foi responsável por $45 \%$ de todas as parições ocorridas, a diferença de $10,8 \%$ na taxa de parição representa acréscimo importante no número de ninhadas produzidas. Incrementos semelhantes, de 11,1 e 10,5\% na taxa de parição conseguiram-se com coelhas lactantes separadas de suas ninhadas, respectivamente, por 40 (Maertens, 1998) e 36 horas (Alvariño et al., 1998) 
antes da inseminação. Resultados parcialmente contrastantes a estes, obtidos por Virág et al. (1999), incluíram elevação na taxa de parição apenas em primíparas, dentre fêmeas de primeira à sexta lactações, bioestimuladas e inseminadas no dia 11 pós-parto. Os autores atribuíram a baixa taxa de parição das coelhas primíparas do grupo controle, de apenas $18,6 \%$ contra $61,5 \%$ no grupo tratado, à utilização de coelhas neozelandezas puras, e não mestiças.

Theau-Clement \& Boiti (1998) julgaram três hipóteses para explicar o efeito benéfico da separação da ninhada sobre atividade reprodutiva na coelha: 1) breve redução na secreção de prolactina e conseqüente supressão temporária de seu suposto efeito antagônico sobre a liberação de gonadotrofinas; 2) estímulo da ocitocina às contrações uterinas favorecendo o transporte de espermatozóides até o local de fertilização, caso a amamentação ocorresse imediatamente após a inseminação (ou cobrição); 3) a separação mãe-ninhada agiria como um fator "estressante positivo", influenciando o equilíbrio hormonal da coelha. Há evidência experimental, em vacas de corte e ratas, de que o estímulo da amamentação em si, é o responsável pela supressão da secreção de gonadotrofinas, e não as altas concentrações de prolactina associadas à lactação (Williams, 1990).

Além da ordem de lactação, outros fatores devem ser considerados ao se comparar resultados de pesquisas, tais como: uso de inseminação artificial ou

Tabela 1 - Receptividade sexual e taxa de parição em coelhas lactantes dos grupos controle e bioestimulado, por categoria de ordem de lactação

Table 1 - Sexual receptivity and kindling rate of lactating rabbit does of the control and bioestimulated groups, by lactation order class

Característica

Trait

Grupo
Group

\section{Controle}

Control

Número de coelhas exposta
Number of exposed does
Receptividade sexual, \%
Sexual receptivity
Taxa de parição, \%
Kindling rate

Número de coelhas expostas

Number of exposed does

Receptividade sexual, \%

Sexual receptivity

Taxa de parição, \%

Kindling rate

Número de coelhas expostas

Number of exposed does

Receptividade sexual, \%

Sexual receptivity

Taxa de parição, \%

Kindling rate

Número de coelhas expostas

Number of exposed does

Receptividade sexual, \%

Sexual receptivity

Taxa de parição, \%

Kindling rate

87,9

57,6
78

93,6

70,1

103

92,2

59,0

92,1

62,9
Probabilidade Probability

Bioestimulado

Bioestimulated

Primeira lactação

First lactation

$33 \quad 32$

87,5

0,963

53,1

0,718

Segunda à quarta lactações

Second to fourth lactations
81

92,6

68,8

0,804

0,851

Quinta lactação em diante

Fifth lactation and on

88

98,9

0,031

69,8

0,127

Total

201

94,5

0,316

66,7

0,421 
monta natural, utilização de fêmeas puras ou mestiças, temperatura ambiente, programa de luz e plano nutricional. $\mathrm{O}$ estímulo à atividade reprodutiva tende a ser inócuo em fêmeas subnutridas, ou em balanço nutricional negativo. Esta observação poderia estar relacionada à resposta mais favorável das coelhas de ordens de lactação mais avançadas ao tratamento, visto que o balanço energético de coelhas primíparas é fortemente negativo ao final da primeira lactação (Parigi-Bini et al., 1996).

As médias gerais e os desvios-padrão do número de nascidos totais e nascidos vivos por ninhada, taxa de natimortalidade e peso da ninhada ao nascer referentes às ninhadas seguintes $(\mathrm{N}=264)$ foram, respectivamente: $7,02 \pm 3,25 ; 5,59 \pm 3,49 ; 0,272 \pm 0,336$; e 404,3 $\pm 224,6$ g. Nenhuma dessas características foi influenciada pelo tratamento, mas todas, pelo mês de cobrição da fêmea $(\mathrm{P}<0,05)$. $\mathrm{O}$ efeito do mês pode, em parte, ser atribuído à variação estacional da temperatura e outros fatores ambientais. Alvariño et al. (1998) e Castellini et al. (1998) também não detectaram diferenças entre fêmeas controle e bioestimuladas, quanto ao desempenho da ninhada seguinte ao nascer. Já Maertens (1998) e Szendro et al. (1999) relataram aumento no tamanho da ninhada ao nascer, em fêmeas lactantes bioestimuladas. Encontraram diferenças no número de nascidos totais, favorecendo as fêmeas do grupo bioestimulado, apenas no pico de produção, ou seja, da segunda à quinta parições.

As médias gerais, desvios-padrão e valores extremos das características de desempenho coletivo e individual à desmama e aos 70 dias de idade das ninhadas separadas (ou não) são apresentados na Tabela 2. Ocorreu a morte de 10 fêmeas durante o

Tabela 2 - Estatísticas descritivas das características de desempenho das ninhadas separadas (ou não) das mães e das ninhadas seguintes ao nascer

Table 2 - Descriptive statistics of performance traits of litters separated (or not) from their mothers and of the next litters at birth

\begin{tabular}{|c|c|c|c|c|c|}
\hline Característica & Número de observações & Média & $\mathrm{DP}$ & Mínimo & Máximo \\
\hline Trait & Number of observations & Mean & $S D$ & Minimum & Maximum \\
\hline
\end{tabular}

Desempenho das ninhadas separadas Performance of separated litters

Número de nascidos

Number born

Número de lactentes ${ }^{1}$

Number of suckling kits ${ }^{1}$

Número de desmamados

Number weaned

Número aos 70 dias de idade

Number at 70 days of age

Peso total da ninhada à desmama $(\mathrm{g})$

Litter weaning weight

Peso médio da ninhada à desmama $(\mathrm{g})$

Mean litter weaning weight

Peso total da ninhada aos 70 dias (g)

Litter weight at 70 days

Peso médio da ninhada aos 70 dias (g)

Mean litter weight at 70 days

Taxa de mortalidade da separação à desmama

Mortality rate from separation to weaning

Taxa de mortalidade da separação aos 70 dias

Mortality rate from separation to 70 days

Peso individual à desmama $(\mathrm{g})$

Individual weaning weight

Peso individual aos 70 dias (g)

Individual weight at 70 days

Ganho de peso médio diário da desmama aos 70 d (g/dia)

Average daily gain from weaning to 70 days (g/day)

$\begin{array}{lrrrc}414 & 7,68 & 2,18 & 4 & 11 \\ 414 & 7,16 & 1,85 & 4 & 11 \\ 414 & 6,37 & 1,93 & 0 & 11 \\ 413 & 6,04 & 1,91 & 0 & 10 \\ 413 & 6.021 & 1.619 & 0 & 9.950 \\ 413 & 953,8 & 154,9 & 0 & 1.307,5 \\ 411 & 12.227 & 3.789 & 0 & 21.035 \\ 411 & 2.018,6 & 276,2 & 0 & 2.585,0 \\ 414 & 0,11 & 0,17 & 0 & 1,00 \\ 413 & 0,15 & 0,18 & 0 & 1,00\end{array}$

Desempenho individual dos láparos das ninhadas separadas Individual performance of kits of separated litters

$\begin{array}{lrrrr}2.618 & 950,3 & 155,9 & 300,0 & 1.450,0 \\ 2.459 & 2.048 & 231 & 1.075 & 2.735 \\ 2.459 & 31,73 & 5,13 & 4,71 & 54,29\end{array}$

${ }^{1}$ No dia da separação da mãe (The day of separation). 
experimento, quatro do grupo tratado. Registrou-se perda total de quatro ninhadas antes da desmama, das quais três pertenciam ao grupo tratado. Estas ninhadas receberam o índice zero para peso total e médio da ninhada. Assim, as diferenças entre o número total de apresentações (415) e os números de observações referentes ao desempenho de ninhadas apresentados na Tabela 2 são consequiências do extravio de dados.

Não se notou diferença no número de láparos por ninhada antes da separação entre os grupos. Não se detectou efeito $(\mathrm{P}>0,05)$ da interação tratamento $\mathrm{x}$ ordem de lactação, ou do tratamento isoladamente, sobre o número de láparos por ninhada à desmama ou aos 70 dias de idade. Esses resultados concordam, parcialmente, com os de Virág et al. (1999), que não observaram efeito de 48 horas de separação da mãe, no décimo primeiro dia de lactação, sobre o tamanho das ninhadas aos 21 dias de idade. Também TheauClement \& Mercier (1999) não encontraram efeito de 24 horas de separação, no mesmo dia, sobre o tamanho da ninhada à desmama, com 28 dias de idade.

Houve efeito da interação tratamento $\mathrm{x}$ ordem de lactação sobre a taxa de mortalidade da separação até os 70 dias de idade $(\mathrm{P}=0,021)$, e tendência de efeito desta interação, sobre a taxa de mortalidade da separação até a desmama $(\mathrm{P}=0,058)$. Tendo em vista a importância das taxas de mortalidade em cunicultura, analisou-se o efeito do tratamento, dentro de cada categoria de ordem de lactação, para estas duas características (Tabela 3). Diferenças importantes entre os grupos quanto às taxas de mortalidade foram observadas apenas em ninhadas

Tabela 3 - Médias de quadrados-mínimos (e erros-padrão) dos pesos total e médio da ninhada à desmama e aos 70 dias de idade, nos grupos controle e bioestimulado, por categoria de ordem de lactação

Table 3 - Least squares means (and standard errors) of total and average litter weaning and 70-day weights in the control and bioestimulated groups, by lactation order class

\begin{tabular}{lc}
\hline Característica & Controle \\
Trait & Control \\
\hline
\end{tabular}

Número de ninhadas antes da separação

Number of litters prior to separation

Taxa de mortalidade da separação até a desmama

Mortality rate from separation to weaning

Taxa de mortalidade da separação até os 70 dias

Mortality rate from separation to 70 days

Número de ninhadas antes da separação

Number of litters prior to separation

Taxa de mortalidade da separação até a desmama

Mortality rate from separation to weaning

Taxa de mortalidade da separação até os 70 dias

Mortality rate from separation to 70 days

Número de ninhadas antes da separação

Number of litters prior to separation

Taxa de mortalidade da separação até a desmama

Mortality rate from separation to weaning

Taxa de mortalidade da separação até os 70 dias

Mortality rate from separation to 70 days

Número de ninhadas antes da separação

Number of litters prior to separation

Taxa de mortalidade da separação até a desmama

Mortality rate from separation to weaning

Taxa de mortalidade da separação até os 70 dias

Mortality rate from separation to 70 days
33

$0,098(0,045)$

$0,120(0,043)$

78

$0,117(0,019)$

$0,158(0,019)$

102

$0,093(0,014)$

$0,140(0,017)$

213

$0,116(0,014)$

$0,154(0,014)$
Bioestimulado

Bioestimulated

Primeira lactação

First lactation

$$
32
$$

$$
0,200(0,049)
$$

0,118

$0,248(0,047)$

0,046

Segunda à quarta lactações Second to fourth lactations

81

$$
\begin{array}{ll}
0,107(0,018) & 0,725 \\
0,142(0,018) & 0,546
\end{array}
$$

Quinta lactação em diante Fifth and further lactations

\section{8}

$$
\begin{gathered}
0,103(0,015) \\
0,146(0,018) \\
\text { Total } \\
201
\end{gathered}
$$

0,828

R. Bras. Zootec., v.32, n.2, p.315-324, 2003 
de fêmeas primíparas. Nesta categoria, tanto a taxa de mortalidade da separação até a desmama como a da separação até os 70 dias de idade corresponderam, no grupo bioestimulado, a mais que o dobro que as do grupo controle, embora a diferença não tenha sido significativa no primeiro caso $(\mathrm{P}=0,118)$. É fato bem documentado, em coelhas, a menor produção de leite na primeira lactação, resultando em ninhadas mais leves à desmama (Lukefahr et al., 1983; Singh, 1996; Moura et al., 2000). Assim, a separação temporária da mãe, no caso das primíparas, pode ter retardado ainda mais o desenvolvimento da ninhada, aumentando, inclusive, a taxa de mortalidade a partir da separação. Da segunda lactação em diante, as diferenças entre grupos foram muito menores e não significativas, evidenciando que as ninhadas de coelhas multíparas não apresentam diferenças, devidas ao tratamento, nas taxas de mortalidade da separação até a desmama ou até os 70 dias de idade (Tabela 3).

A separação da mãe influenciou adversamente os pesos totais e médios da ninhada à desmama, independentemente da categoria de ordem de lactação. Todavia, este efeito não perdurou até os 70 dias de idade (Tabela 4). As diferenças entre grupos de $469 \mathrm{~g}(7,5 \%)$ no peso total e de $49,5 \mathrm{~g}(5,1 \%)$ no peso médio da ninhada à desmama, foram reduzidas para $349 \mathrm{~g}(2,8 \%)$ e $40 \mathrm{~g}(1,9 \%)$, respectivamente, aos 70 dias de idade. Conforme demonstrado por Szendro et al. (1999), além da perda de uma mamada, ocorre redução na produção de leite nos dias subseqüentes à separação entre mãe e ninhada, contribuindo para reduzir o peso à desmama. Estes autores estudaram o efeito da privação de uma mamada em torno do décimo dia de lactação, sobre a produção de leite das coelhas e auferiram redução de 33,15 e $6 \%$ no grupo tratado, respectivamente, nos primeiros três dias após o tratamento. De acordo com seus resultados, os pesos dos láparos perdedores de uma mamada foi inferior aos do grupo controle, da separação (dia 11) até os 70 dias de idade.

Os resultados referentes ao desempenho individual dos láparos à desmama e aos 70 dias são consistentes com o desempenho coletivo das ninhadas (Tabela 4). Da mesma forma que o peso médio, o peso individual à desmama foi reduzido em cerca de 5\% pela separação da mãe, porém, a diferença tornou-se muito pequena e não significativa aos 70 dias de idade. Interessantemente, o ganho de peso médio diário da desmama aos 70 dias foi maior no grupo bioestimulado, fornecendo evidência de ganho

Tabela 4 - Médias de quadrados-mínimos (e erros-padrão) dos pesos individuais e da ninhada à desmama e aos 70 dias de idade nos grupos controle e bioestimulado

Table 4 - Least squares means (and standard errors) of individual and litter weights at weaning and at 70 days in the control and bioestimulated groups

\begin{tabular}{|c|c|c|c|}
\hline $\begin{array}{l}\text { Característica } \\
\text { Trait }\end{array}$ & $\begin{array}{l}\text { Controle } \\
\text { Control }\end{array}$ & $\begin{array}{l}\text { Bioestimulado } \\
\text { Bioestimulated }\end{array}$ & $\begin{array}{l}\text { Probabilidade } \\
\text { Probability }\end{array}$ \\
\hline Número de ninhadas antes da separação & 213 & 201 & \\
\hline $\begin{array}{l}\text { Number of litters prior to separation } \\
\text { Peso total da ninhada à desmama }(\mathrm{g}) \\
\text { Litter weaning weight }\end{array}$ & $6.222(89)$ & $5.753(93)$ & 0,004 \\
\hline $\begin{array}{l}\text { Peso médio da ninhada à desmama }(\mathrm{g}) \\
\text { Mean litter weaning weight }\end{array}$ & $978,4(7,7)$ & $928,9(8,1)$ & 0,001 \\
\hline $\begin{array}{l}\text { Peso total da ninhada aos } 70 \text { dias (g) } \\
\text { Litter weight at } 70 \text { days }\end{array}$ & $12.485(184)$ & $12.136(194)$ & 0,340 \\
\hline $\begin{array}{l}\text { Peso médio da ninhada aos } 70 \text { dias }(\mathrm{g}) \\
\text { Mean litter weight at } 70 \text { days }\end{array}$ & $2.063(13)$ & $2.023(14)$ & 0,095 \\
\hline Número de indivíduos à desmama & 1.365 & 1.253 & \\
\hline $\begin{array}{l}\text { Number of individuals at weaning } \\
\text { Peso individual à desmama (g) } \\
\text { Individual weaning weight }\end{array}$ & $962,7(4,5)$ & $912,4(4,9)$ & 0,001 \\
\hline $\begin{array}{l}\text { Número de indivíduos aos } 70 \text { dias } \\
\text { Number of individuals at } 70 \text { days }\end{array}$ & 1.280 & 1.179 & \\
\hline $\begin{array}{l}\text { Peso individual aos } 70 \text { dias (g) } \\
\text { Individual weight at } 70 \text { days }\end{array}$ & $2.049,9(7,6)$ & $2.022,8(8,4)$ & 0,170 \\
\hline $\begin{array}{l}\text { Ganho de peso médio diário da desmama aos } 70 \text { dias }(\mathrm{g} / \mathrm{d}) \\
\text { Average daily gain from weaning to } 70 \text { days }\end{array}$ & $31,44(0,17)$ & $32,25(0,19)$ & 0,049 \\
\hline
\end{tabular}

R. Bras. Zootec., v.32, n.2, p.315-324, 2003 
compensatório neste período (Tabela 4). Embora não tenham acompanhado o desempenho dos láparos no período pós-desmame, Theau-Clement \& Mercier (1999) relataram a expectativa de ganho compensatório no período de engorda, devido à ligeira compensação por eles detectada já no final do período prédesmame. Szendro et al. (1999) encontraram diferenças no peso dos láparos entre grupo controle e tratado que se reduziram de 7,2\% (26 g) aos 21 dias para apenas 1,2\% (34 g) aos 70 dias de idade. Mesmo assim, os autores concluíram que os láparos não foram capazes de compensar inteiramente a diferença, porque esta continuava (estatísticamente) significativa aos 70 dias. No entanto admitiram que o atraso no abate, de apenas um dia, não foi substancial.

Em resumo, a bioestimulação apresentou efeito favorável sobre a receptividade e taxa de parição de fêmeas de ordem de lactação mais avançada. O efeito da separação temporária da mãe sobre as ninhadas diferiu entre coelhas primíparas e multíparas: a taxa de mortalidade dos láparos da separação aos 70 dias de idade foi maior para as primeiras. Quanto aos pesos total e médio da ninhada e individual, as diferenças existentes à desmama foram, em grande parte, compensadas durante o período de crescimento pós-desmama, tornando-se pouco importantes aos 70 dias de idade. Sob o regime de monta natural, ainda adotado em larga escala em cunicultura, a separação mãe-ninhada pode significar economia importante de tempo de trabalho com as cobrições de coelhas lactantes. Seria importante incluir, se possível, a variável "tempo despendido com a cobrição assistida" nos futuros estudos.

\section{Conclusões}

Pode-se recomendar a separação temporária entre mãe e ninhada, entre o sétimo e o nono dias pósparto, no período de 40 horas que antecede a cobrição, para melhorar a receptividade e o desempenho reprodutivo de coelhas multíparas, especialmente da quinta lactação em diante. $\mathrm{O}$ menor peso à desmama é compensado com maior velocidade de ganho no período pós-desmame, sem que haja aumento na taxa de mortalidade. A exceção são as coelhas primíparas cujo manejo deve ser diferenciado, evitando-se a separação temporária de suas ninhadas porque eleva substancialmente a taxa de mortalidade dos láparos.

\section{Agradecimento}

À Sra. Irene Francisca de Arruda e às alunas do curso de Graduação em Zootecnia Elisângela Aparecida Guaiume e Elisabete Okuda Yamaguishi, pelo apoio técnico, e ao Professor Pedro Fernandes, pela revisão de língua portuguesa.

\section{Literatura Citada}

ALVARIÑO, J.M.R; UBILLA, E.. Fisiología de la Reproducción en la Hembra. In: ALVARIÑO, M.R. (Ed.) Control de la reproducción en el conejo. Madrid: Ediciones MundiPrensa, 1993. p.33-50.

ALVARIÑO, J.M.R.; DEL ARCO, J.A.; BUENO, A. Effect of mother-litter separation on reproductive performance of lactating rabbit females inseminated on day 4 or 11 post partum. World Rabbit Science, v.6, n.1, p.191-194, 1998.

BOITI, C. International collaboration in rabbit reproduction research: presentation of the IRRG group. World Rabbit Science, v.6, n.1, p.175-178, 1998.

BOITI, C.; CASTELLINI, C.; CANALI, C. et al. Long term effect of PMSG on rabbit does reproductive performance. World Rabbit Science, v.3, n.2, p.51-56, 1995.

CASTELLINI, C. Gestione della riproduzione nelle fattrici cunicole. Rivista di Coniglicoltura, v.12, p.21-27, 1995.

CASTELLINI, C. Recent advances in rabbit artificial insemination. In: WORLD RABBIT CONGRESS, 6., 1996, Toulouse. Anais... Toulouse: World Rabbit Science, 1996. v.2, p.13-26.

CASTELLINI, C.; CANALI, C.; BOITI, C. Effect of motherlitter separation for 24 hours, by closing the nestbox or change of cage, on rabbit doe reproduction performance. World Rabbit Science, v.6, n.1, p.199-203, 1998.

LEBAS, F.; COUDERT, P.; de ROCHAMBEAU, H. et al. Reproduccion. In: LEBAS, F. (Ed.) El conejo: cría y patologia Roma: Organização das Nações Unidas para a Agricultura e Alimentação (FAO), 1996. 227p.

LUIZI, F.; CRIMELLA, C. Effect of change of cage 2 days before artificial insemination on reproductive performance of rabbit does. World Rabbit Science, v.6, n.1, p.195-198, 1998.

LUKEFAHR, S.; HOHENBOKEN, W.D.; CHEEKE, P.R. Characterisation of straightbred and crossbred rabbits for milk production and associative traits. Journal Animal Science, v.57, p.1100-1107, 1983.

MAERTENS, L. Effect of flushing, mother-litter separation and PMSG on the fertility of lactating does and the performance of their litter. World Rabbit Science, v.6, n.1, p.185-190, 1998.

MAERTENS, L., LUIZI, F.; GRILLI, G. Effects of PMSG induced oestrus on the performance of rabbit does: a review. World Rabbit Science, v.3, p.191-194, 1995.

MOURA, AS.A.M.T.; POLASTRE, R. Tendência genética direta e materna de características de desempenho individual em coelhos do grupo genético Botucatu. In: REUNIÃO ANUAL DA SOCIEDADE BRASILEIRA DE ZOOTECNIA, 37., 2000, Viçosa, MG. Anais... São Paulo: Sociedade Brasileira de Zootecnia/Gnosis, [2000] CD-ROM. Melhoramento Animal. 0007. 
MOURA, A.S.A.M.T.; POLASTRE, R; WECHSLER, F.S. Dam and litter inbreeding and environmental effects on litter performance in Botucatu rabbits. World Rabbit Science, v.8, n.4, p.151-158, 2000.

PARIGI-BINI, R.; XICCATO, G.; DALLE-ZOTTE, A. et al. Effect of remating interval and diet on the performance and energy balance of rabbit does. In: WORLD RABBIT CONGRESS, 6., 1996, Toulouse. Anais... Toulouse: World Rabbit Science Association, 1996. p.253-258.

RUSSO, C.; PREZIUSO, G.; PACI, G. et al. Effetto della linea paterna, dell'età di macellazione e del sesso sul profilo acidico della carne di coniglio. Rivista di Coniglicoltura, v.35, n.1, p.29-32, 1998.

SAMPAIO, I.B.M. Estatística aplicada à experimentação animal.Belo Horizonte: Fundação de Ensino e Pesquisa em Medicina Veterinária, 1998. 221p.

SAS INSTITUTE. User's procedures guide. Version 6.11, 4.ed. v.2, Cary: 1996. 842p.

SINGH, G. Genetic and non-genetic factors affecting milk yield of rabbit does under hot semi-arid climate. World Rabbit Science, v.4, n.2, p.79-84, 1996.

SZENDRO, Z.; JOVANCZAI, ZS.; THEAU-CLEMENT et al. The effect of doe-litter separation on production performance of does and their kits. World Rabbit Science, v.7, n.3, p.165-169, 1999.

THEAU-CLEMENT, M.; BOITI, C. Bioestimulation methods for breeding rabbit does: synthesis of the first results. World Rabbit Science, v.6, n.1, p.205-208, 1998.
THEAU-CLEMENT, M.; CASTELLINI, C.; MAERTENS, L. et al. Bioestimulations applied to rabbit reproduction: theory and practice. World Rabbit Science, v.6, n.1, p.179-184, 1998.

THEAU-CLEMENT, M.; MERCIER, P. Effect of a 24 hour doelitter separation on rabbit doe reproductive performance and growth of the young. World Rabbit Science, v.7, n.3, p.177179, 1999.

VIRÁG, GY., KUSTOS, K., SZABÓ, L. Effect of a 48 hours doe litter separation on rabbit doe reproductive performance and offspring's growth. World Rabbit Science, v.7, n.3, p.155159, 1999.

WALTER, M.R.; MARTINET, L.; MORET, B. et al. Régulation photepériodique de láctivité sexuelle chez le lapin mâle et femelle. Archives d'Anatomie d'Histologie et d'Embryologie, v.51, p.773-780, 1968.

WILLIAMS, G.L. Suckling as a regulator of postpartum rebreeding in cattle: a review. Journal of Animal Science, v.68, n.3, p.831-852, 1990. 